\title{
A new conceptual design method to support rapid and effective mapping from product design specification to concept design
}

\author{
Haizhu Zhang ${ }^{1}$ - Xin $\mathrm{Han}^{1} \cdot \operatorname{Rong} \mathrm{Li}^{1} \cdot$ Shengfeng Qin ${ }^{2}$ - Guofu Ding ${ }^{1} \cdot$ Kaiyin Yan $^{1}$
}

Received: 3 December 2015 / Accepted: 1 March 2016 /Published online: 28 March 2016

(C) The Author(s) 2016. This article is published with open access at Springerlink.com

\begin{abstract}
Conceptual design has a decisive impact on the product development time, cost and success. This paper presents a new conceptual design method for achieving rapid and effective mapping from product design specification (PDS) to concept design. This method can guide the creation of reasonable mapping among the PDS, behaviour parameters and structure parameters and to evaluate the rationality of performance parameters and structure parameters to confirm a reasonable conceptual design scheme. In this method, we establish a PDS-behaviour-structure conceptual design model to support the conceptual design of multi-disciplinaryoriented complex product system (CoPS) and develop a vector-based mapping tool in this method to support the rapid mapping, and demonstrate its feasibility and effectiveness by a case study. This method is not only supportive to realise the automation of a conceptual design process but also helpful to evaluate the conceptual design in the field of engineering design.
\end{abstract}

Keywords Conceptual design model · Vector mapping · Complex product systems $\cdot$ Multi-disciplinary $\cdot$ Product design specification

Shengfeng Qin

sheng-feng.qin@northumbria.ac.uk

Guofu Ding

dingguofu@163.com

1 Institute of Advanced Design and Manufacturing, Southwest Jiaotong University, Chengdu 610031, People's Republic of China

2 School of Design, Northumbria University, Newcastle upon Tyne NE1 8ST, UK

\section{Introduction}

Pahl and Beitz split the design process into four main phases, which are requirement analysis, conceptual design, layout design and detail design [1]. Conceptual design has a decisive impact on the product development time, cost and success. The essential tasks of conceptual design are to figure out product design specification (PDS), establish function structures and search for appropriate principle solutions and synthesise these into a product conceptual design scheme. A conceptual design process is composed of design analysis and design synthesis [2]. Design analysis is to break up a whole complex product system (CoPS) into its elements (decomposition) and then study these elements and their interrelationships; design synthesis is to integrate all parts or elements to produce new systematic effects [3]. In the product requirement analysis stage, subjective and fuzzy customer requirements are transformed into objective and accurate PDS as the input of a conceptual design. PDS typically contains the design inputs, design constraints and design goals and a list of the product performance, environment, quality, reliability, security, life cycle and other elements [4]. Therefore, how to quickly and effectively transfer the PDS elements and map them onto a design scheme (or solution) is crucially challenging in conceptual design.

According to the $\mathrm{V}$ model [5], generating a conceptual design scheme from iterative decomposition and synthesis of a CoPS is an iterative process. Especially, there are many multi-disciplinary and multi-field coupling relationships of CoPS in functions, subsystems and components [6] and many-to-many mapping relationships between functions and structures [7], so it is difficult to form a reasonable conceptual design scheme by applying a system design or an axiomatic design theory [8] alone. In fact, a CoPS features not only the functional diversity, complex hierarchy of functionalities and 
performance influenced by a dynamic environment but also the complicated performance requirements. The performance requirements have a significant effect on functional decomposition, behaviour mapping and structure selection. Thus, at the conceptual design stage of CoPS, it needs to properly and systematically consider and analyse the performance requirements and the coupling relationships among the product functions and structures caused by interdisciplinary and multi-field design spaces. Otherwise, the resultant design scheme is more likely to be a defective design scheme, possibly failing to meet customer demand and generating more iterations of the whole design activities [9]. In other words, we need to create reasonable mapping from PDS to a conceptual design behaviour and structure scheme and to evaluate the mapping result reasonably. For example, the conceptual design of a high-speed train's braking system involves collaboration of mechanics, control, pneumatics and other disciplines. Its system structure contains the braking control system, air supply system, basic brake device, brake disc and so on, so it has a typical multidisciplinary and multi-level structure of CoPS. In order to obtain an effective and safe conceptual design scheme of such a braking system, it is necessary to collaborate with experts in various disciplines to analyse the interactions of multidisciplinary behaviours and structure parameters.

In this paper, we propose a vector-based mapping method to support the reasonable mapping among the PDS, behaviour parameters and structure parameters, and rational evaluation of performance parameters and structure parameters. Based on this, we establish a new conceptual design model to support the conceptual design of multi-disciplinary-oriented CoPS. Our contributions have twofold:

(1) We propose a PDS-behaviour-structure (P-B-S) conceptual design model to support multi-disciplinary collaborative conceptual design of CoPS. It describes conceptual design from three dimensions: the structure, discipline and domain. When applying this model in conceptual design, firstly, based on the design objectives and design constraints of PDS, a qualitative evaluation of the conceptual design functions is implemented. Secondly, oneto-many mapping of a function to behaviours is realised so that the qualitative and quantitative behaviour descriptions of CoPS are formed. Finally, it is to realise the mapping from behavioural variables to structure parameters and then analyse multidisciplinary behaviours and to evaluate the rationales of structural parameters for forming a feasible conceptual design scheme. In this way, the conceptual design process of CoPS is well structured, leading to a rapid conceptual design.

(2) We advise a vector-based mapping tool to support conceptual design analysis and synthesis process. It consists of a vector to express design elements and a mapping matrix to describe relationships between the elements and possible design responses. In order to improve the efficiency of iterative product design processes, firstly, we form a PDS vector, in the order of the degree of importance of PDS parameters which is obtained by taking into account the subjective and objective factors of the conceptual design. Then, we follow the proposed PB-S conceptual design model to create corresponding mapping matrices to describe mapping relationships among the PDS, behaviour and structure. We also establish a disciplinary behaviour matrix (DBM) to express the relationships between different disciplines and behaviour parameters and to evaluate the behaviour and structure. The establishment of a mapping matrix needs some expert knowledge support. With this vector-based mapping tool, we can narrow down the design exploration spaces quickly guided by the mapping matrices and effectively evaluate the design schemes.

The rest of the paper is organised as follows: Section 2 summarises some previous related work and research methods, which mainly include conceptual design modelling, solutions and tools. Section 3 introduces the P-B-S conceptual design model and the vector-based mapping tools. Section 4 takes the conceptual design of a high-speed train's braking system as an example to verify the usefulness and effectiveness of the proposed method, and finally, the conclusions and future work are drawn in Section 5.

\section{Related work}

Conceptual design was firstly mentioned in the monograph of engineering design [11]. The fundamental objective of basic theoretical research of conceptual design is to generate a conceptual design scheme and to evaluate the rationale (or correctness) of the scheme. Recently, researchers have put forward some design models, methods and tools to support the conceptual design. These researches concentrate on three aspects: (1) studying acquisition and expression of product conceptual design knowledge, which focuses on establishing the model of product conceptual design information and knowledge, namely modelling problem; (2) choosing appropriate methods to solve the established product information model and to generate a conceptual design scheme, namely reasoning problem; and (3) studying a conceptual design generation tool to assist designers in achieving a rapid and effective conceptual design, namely tool problem. Formal models and solving methods of conceptual design are the basis of building a rapid and effective conceptual design tool. The review on related works is as follows. 


\subsection{Conceptual design modelling}

There are multiple representation methods of a product information description model, such as language, geometry model, graph, tree, object, knowledge model and so on [12], of which information description based on knowledge has become one hotspot in this field in recent years, since it can fully describe the conceptual design information and effectively support the generation of conceptual design scheme. Additionally, it is easy to express and process on the computer. Knowledge categories of conceptual design involve many aspects, such as customer requirement, product function, performance, specification, behaviour, abstract structure and so on [13]. Conceptual design knowledge representation expresses the knowledge acquired during the early design stages via the use of symbols and methods accepted and processed by computers, which is the basis of the research on the design model and conceptual design. In order to support conceptual design scheme reasoning, knowledge of conceptual design involves not only product cases but also the designer's experience, reasoning rules, etc. Chen et al. [10] proposed a conceptual design knowledge framework based on function specification and information flow. Based on the function-behaviourstructure model, Christophe et al. [14] added the customer requirement knowledge and constructed the knowledge ontology representation model of conceptual design. Chang et al. [15] conducted a research on ontology-knowledge representation for product design.

Constructing a conceptual design model and a design process model aims to standardise conceptual design activities and provide a paradigm, model and guidance framework for the design process, which makes the design process standardised, structuralised and computable. Finger and Dixon [16] conducted the earliest research and put forward four models including descriptive model, cognitive model, prescription model and computable model to describe design activity and their physical principles, while Cross [17] suggested describing the process model of design activity with descriptive model and expository model. It is now generally agreed that essential characteristics of the extended model based on functional design are listed as follows. Firstly, convert design requirement into functional requirement and then establish functional hierarchy to express the whole system and finally acquire requirement solutions through the mapping and integration. On the basis of three representative models including function-structure model [18], function-behaviourstate model [19] and function-behaviour-structure model [20], some scholars put forward conceptual design expansion models, such as the function-environment-behaviourstructure model proposed by Deng [21] for the sake of environmental protection and the concept design process model of requirement-function-principle-system proposed by Chen et al. [22]. In recent years, the Design Engineering
Laboratory of the University of Oregon in the USA explored a standardised, structured and computable method supported by a unified definition of functional groups [23] and published a series of research achievements [24]. In the field of conceptual design of CoPS, in addition to the research on the basic theory of conceptual design, recent research directs to describe the conceptual design knowledge system of CoPS with multilevel and flexible product information models. The focus of these researches is the description of CoPS information with multi-level and flexible management of design change information [25].

\subsection{Conceptual design solution}

The process of solving a conceptual design model usually is getting a design concept from a combination of heuristic algorithm and matrix algorithm. The heuristic algorithm can avoid the combinatorial explosions in the solving process by determining a feasible region, reducing solution space with rules and constraints, while the matrix algorithm can map and analyse all kinds of relations between the properties of the products or between the design processes [26]. For example, the customer needs were mapped onto the engineering properties by house of quality [27], and Suh's axiom design [8] defined a design matrix as the correlation (transformation) matrix between related design subdomains. Design matrix and design structure matrix [28] are applied to analyse and optimise coupling relationships among the functions, subsystems and structures via clustering and decomposition algorithms. Based on evolutionary theory, the conceptual design study [29] showed that biological growth is similar to the evolution of product architecture, so that the various evolutionary algorithms can be introduced into product design.

The ultimate goal of conceptual design is to obtain a reasonable design; thus, some scholars use a variety of reasoning technologies in the searching process of design scheme. At present, reasoning methods in conceptual design are for qualitative reasoning, which mainly include case-based reasoning [30], design catalogue [31], rule-based reasoning [32], ontology-knowledge reasoning [33] and artificial reasoning [34].

\subsection{Conceptual design tools}

In recent years, many computer-aided design tools have been developed, but most of the design tools mainly focus on requirement analysis (e.g. quality function deployment), layout design and detailed design stages (e.g. design structure matrices, graph grammars, solid models, dynamic modelling and finite element analysis), which are insufficient for supporting the early product concept design. Although there are many design methods such as brainstorming, internal and external search and morphological analysis to simulate the activities of 
designers, they always rely excessively on the subjective experience of the designers. Based on the existing product design experience and knowledge, Strawbridge et al. [35] proposed a computerisation tool, a concept generation machine, to help designers choose the right components from given functions and to establish a form matrix to calculate the concept variants. At the early stage of design process, it could generate many concepts based on the existing design knowledge. Meanwhile, the concept generation machine could reduce iterations in the design process [36]. Given that the computer-aided product design tools mainly focus on the detailed design, Cardillo et al. [37] proposed a computer-aided innovation system to support a product layout design. Komoto and Tomiyama [38] constructed a computer-aided product architecture design tool based on the function-behaviour-state modelling technology. Bryant et al. [36] developed a method to rapidly generate and arrange a feasible conceptual design scheme from the functional basis of design. Zhang et al. [39] developed a knowledge-based conceptual synthesiser (KBCS) to support a conceptual synthesis of the design process. However, these tools still cannot provide sufficient support for the design of CoPS in terms of conceptual design analysis and synthesis, and the main reason for this is that they do not support the multi-disciplinary analysis and evaluation of conceptual design.

In summary, the following conclusions can be drawn based on analysing the current research status of the conceptual design:

(1) Although the focus of the above researches differs, in fact, the knowledge, modelling and reasoning technology of conceptual design are not completely isolated, but in the trinity. The integral development of three research aspects could lead to a complete conceptual design system. Conceptual design modelling requires effective integration of product design knowledge and design process; if a product conceptual design model is only generated based on the functional transformation and functional mapping, rather than analysing, evaluating and improving the model from the perspective of complex performance requirements, the resulting design scheme must be flawed, and it is difficult to meet customer requirement, in turn causing repetition and iteration of the entire design activities. A good conceptual design model shall cover a variety of product design views and contain a variety of information and a large amount of data, helping the designers get access to the required data easily and quickly and eliminating the redundant data at the same time. However, the expression and description methods of existing conceptual design models lack the ability of reusing the existing design experience and knowledge, ranking of the importance of parameters in the design model and getting rid of redundant parameters so that numerous parameters in a disordered state are involved in the design process, which reduces the design efficiency.

(2) There is a lack of systematic analysis and researches of coupling relationships between product functions and structures from a cross-disciplinary and multi-domain point of view. According to the latest literature search, the recent research suggested (1) to recognise the relationships between the subsystems and components and then (2) to qualitatively analyse and evaluate the conceptual design model. For example, D'Amelio et al. [40] proposed a lining value-filtering and cross-filtering method to identify and predict the possible crossing disciplinary problems in a complex system. Tilstra et al. [41] developed a multi-level design structure matrix to analyse the relationship among the product components and to realise the evaluation and comparison of product structure characteristics.

To sum up, at the conceptual design stage of CoPS, an appropriate method of analysing the coupling relationship of the function and structure and an accurate evaluation of design parameters play a vital role in the whole product development, but this has not yet been well developed.

Here, we propose a new conceptual design method based on the P-B-S conceptual design model and vector-based design mapping tools. This method not only can facilitate the solution to the above problems but also can contribute to realising the rapid and effective mapping from PDS to conceptual design scheme.

\section{A new conceptual design method}

The conceptual design of CoPS is composed of functional decomposition, functional evaluation, behaviour mapping, structure synthesis and so on. In accordance with the V model, there is a parallel and iterative relationship among these stages. Due to the multiple levels of CoPS functions, the relationship between behaviour and structural parameters of the various disciplines is complex and highly coupled. However, research and development of each discipline is relatively independent and lacks of communication and coordination so that it is difficult to evaluate system functions and get a reasonable design scheme [42]. Therefore, in order to effectively support conceptual design iteration and coupling analysis of CoPS, this paper proposes a three-dimensional design space to describe the conceptual design of CoPS, that is, including structure, discipline and domain dimensions. On the structure dimension, a CoPS is described as a system, subsystem and component based on the product structure hierarchy. On the discipline dimension, multi-disciplinary parameters are classified into multi-disciplines, such as mechanics, electronics, 
hydraulics, control and other disciplines. On the domain dimension, according to the idea of the ' $\mathrm{Z}$ ' shape domain mapping of the axiomatic design, design properties are described by the PDS (including performance, function, quality, constraint and so on), behaviour, structure and other domains. Figure 1 shows the multi-dimensional hierarchy space of the conceptual design of CoPS.

This three-dimensional design space provides clear multiple views at different levels of detail of a conceptual design problem in CoPS from its structure, related disciplines and domains. When a CoPS is designed at the system level, for example, a railway line, its related design issues may involve disciplines such as social and economic development planning, local and regional development planning, geography, national and international development strategy and land surveying. Obviously, design and research domains will be corresponding to the disciplines. When a CoPS is designed at the subsystem level, such as a railway vehicle or even a bogie, the involved disciplines could be mechanics, electronics, control, hydraulics, etc.; therefore, the design and research domains will be much more engineering focused such as behaviour (function realisation principles) and structure for engineering integration. When we look at a design at component level, the related disciplines may reduce to a single discipline and the design and research domains could focus on material selections, assembly features, manufacturability, etc.

The conceptual design of CoPS can happen at any level and can be cross-examined at different levels. These three dimensions can still provide an integral design space to explore. In general, at the system level, the main task is to decompose the function of PDS and determine the subfunctions' and metafunctions' principle solutions. Since a function has strong subjectivity, the choice of what physical principles to achieve the function relies on the expert's design experience, so that choices could be qualitatively evaluated with different weights as different conceptual design schemes. At the subsystem level which is the focus of this study, the specific realisation behaviour and structure of function principles are determined and the quantitative description of the conceptual design of CoPS is realised. The behaviour and structure parameters of different disciplines have complex interactions for CoPS. In order to carry out effective management and coordination of the interactions and couplings of different discipline behaviour and structure parameters, in this paper, we propose a PDS-behaviour-structure (P-B-S) conceptual design model. Based on this model, we can categorise the behaviour and structure parameters according to the disciplines and then use a vector-based mapping tool to quantitatively coordinate and analyse the couplings of multi-discipline behaviour and structure parameters of CoPS, thus realising the quantitative evaluation of conceptual design scheme.

\subsection{P-B-S conceptual design model}

Our P-B-S model is developed by extending Gero and Kannengiesser's FBS model [20], integrating top-down decomposition and bottom-up synthesis. For a CoPS, its PDS contains function requirements, performance (including function, quality) requirements, constraints (including environment, human machine, etc.) and so on, the PDS is not only a conceptual design input but also the evaluation criteria of a conceptual design scheme. Thus, in our P-B-S model (see Fig. 2), it is incorporated by replacing the function $(F)$ with the PDS.

The process of conceptual design includes four stages: problem formation, analysis, synthesis and evaluation. The
Fig. 1 Multi-dimensional hierarchy space of the concept design of CoPS

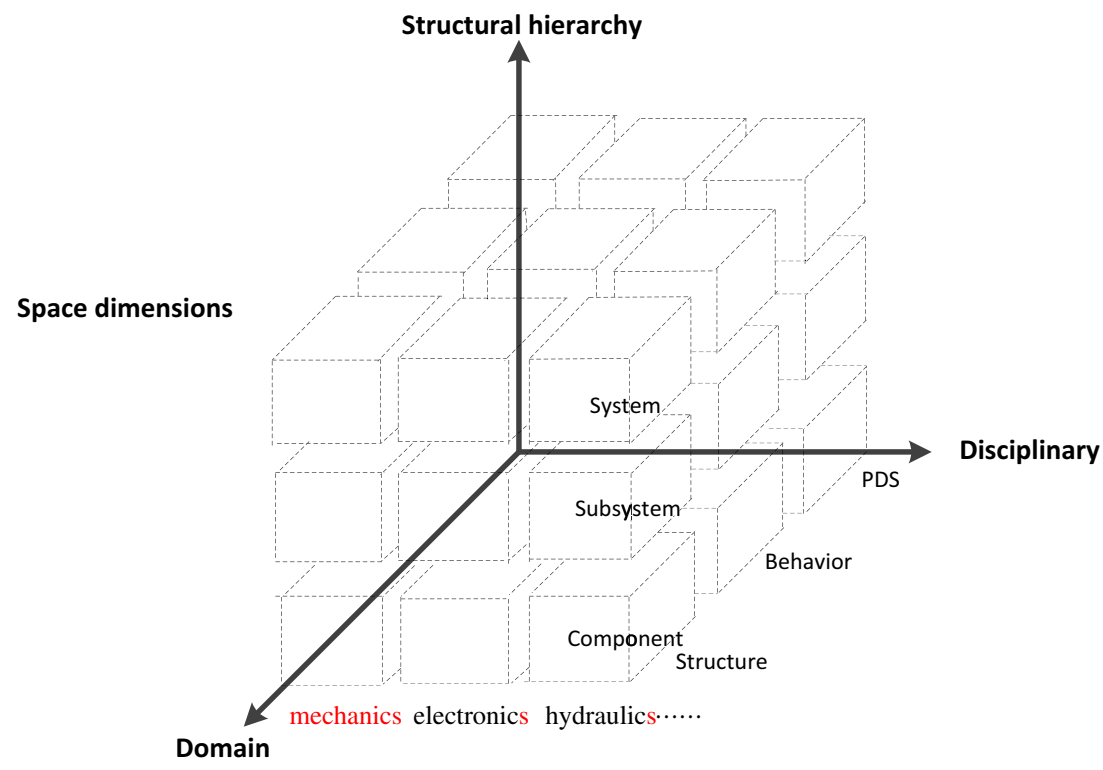




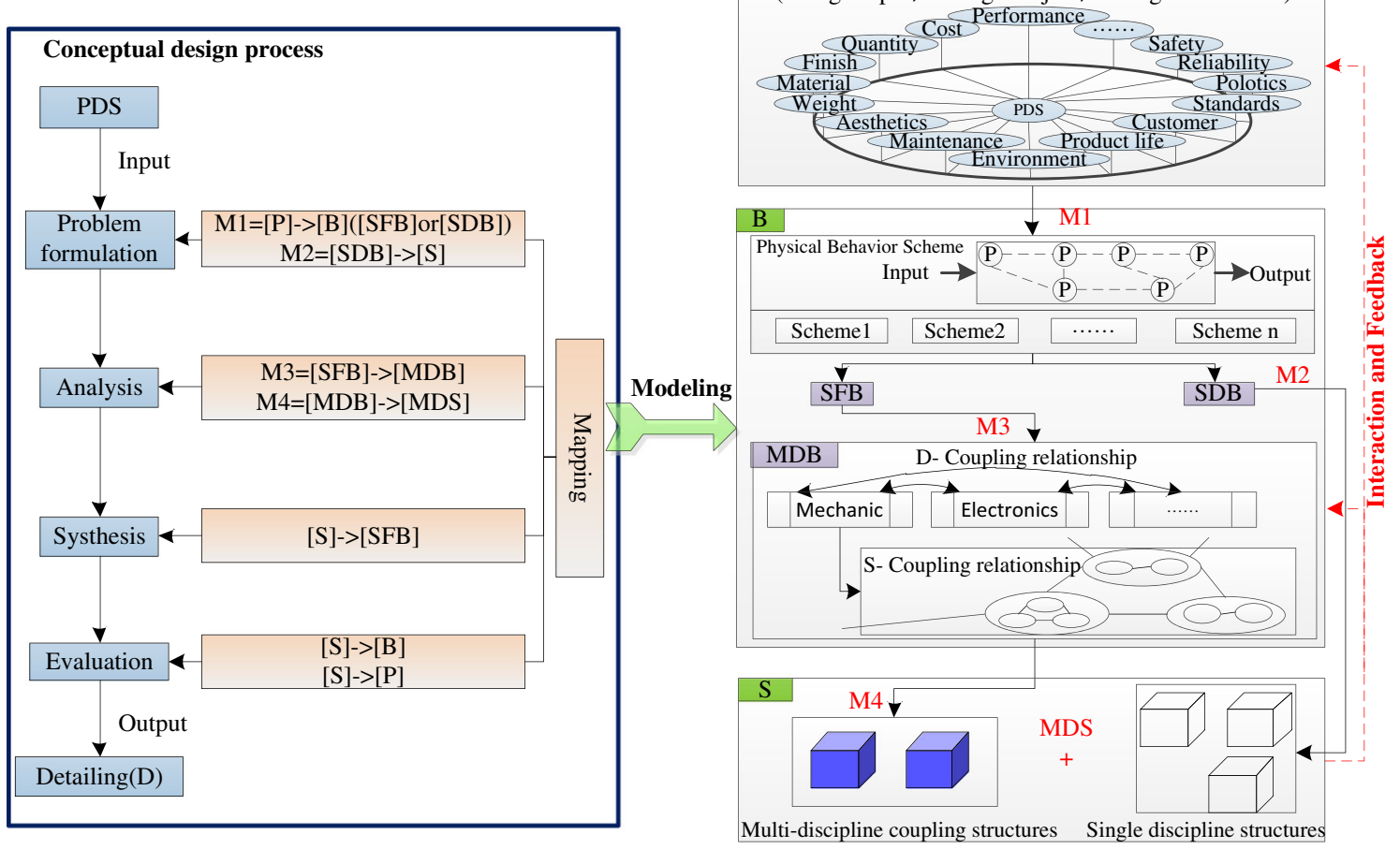

The P-B-S Model

Fig. 2 The P-B-S model

conceptual design starts with PDS as the design boundary. In the formation stage, the functional and non-functional requirements of PDS are analysed and identified to form the design variables and constraints of the conceptual design. At the problem formation stage, there is a need to make the P-B mapping, namely $M_{1}=[P] \geq[B]$. This step is the most creative part of the conceptual design, because for the same function, there are many possible principles to realise it; that is, there are many kinds of functional behaviour schemes. For example, the behaviour scheme could be single discipline based or multi-discipline based. Thus, according to the requirements and constraints in PDS and possible basic physical principles and based on the design experience, we can qualitatively evaluate the CoPS functions at a system level and accordingly determine the principle solutions to meet the requirements, resulting in either a single discipline behaviour (SDB) model or a system function (multi-discipline) behaviour (SFB) model. Therefore, for behaviour $(B)$ in Fig. 2, it includes both of them. When only a single discipline-based behaviour is requested to realise a function(s), a single discipline structure normally can be mapped from $M_{2}$. While a SFB is needed, the coupling mechanism and relationship for creating a multidisciplinary behaviour from possible multi-discipline behaviours (MDBs) and structures need to be explored and evaluated $[43,44]$. The disciplinary behaviour matrix technique can be employed to form a matrix $\left(M_{3}\right)$ for this task. Analysing $M_{3}$ can help explore the practicability of integrating multi- discipline behaviours into a system function behaviour and in turn help decompose a system function behaviour into coupled multi-discipline behaviours and advance the PDSto-SFB mapping to PDS-to-SFB-to-MDB mapping. The practicability evaluation of a coupled MDB scheme will also lead to the mapping of MDB to multi-discipline structure (MDS) via the mapping matrix $\left(M_{4}\right)$. A multidiscipline structure is normally composed of part or whole of single discipline structures (or structure building blocks) and a multi-disciplinary coupling structure to support the realisation of the SFB.

At the analysis stage, the design task is to establish the above mapping matrices by analysing the relationship among the various design elements on the P-B-S model. Once these matrices are developed, they can be evaluated on the basics of practicability. The initial evaluations can lead to new design synthesis solutions at the synthesis stage.

At the evaluation stage, on the basis of synthesis, evaluating whether the system structure scheme can satisfy the requirements of system functional behaviours can be done through the bottom-up approach by comparing the schemed structure and behaviour attributes against the target values and requirements in PDS. This can make the conceptual design process of CoPS a closed loop iterative design process. In the next part, we will explain how the P-B-S design model is expressed and implemented. 


\subsection{Expression and implementation of P-B-S design model}

The construction of the P-B-S design model includes four steps. They are detailed as follows.

\subsubsection{Create a PDS vector}

From a PDS for the conceptual design of CoPS, construct a requirement space. All requirement (specification) elements (or variables) are listed as an initial input vector. Each element can have a weight value to describe its degree of importance. If its weight is 1 , meaning it is a rigid constraint; therefore, we classify it as a design constraint for evaluation of various design schemes. Therefore, the remaining variables with a weighting value between 0 and 1 will be listed orderly in the design requirement space, $P=\left[P_{1}, P_{2}, \ldots, P_{n}\right]$, based on their weightings. The degrees of their importance of a variable can be decided by the subjective or objective experience. The corresponding weightings can form a weighting vector, $W=\left[w_{1}, w_{2}, \ldots, w_{n}\right]$. The resultant vector of $P \times W^{T}$ is called the PDS vector. That is,

$\mathrm{PDS}=P \times W^{T}$

where $P=\left[P_{1}, P_{2}, \ldots, P_{n}\right]$ and $W=\left[w_{1}, w_{2}, \ldots, w_{n}\right]$.Now, we have split the initial PDS into a rigid constraint list $\left[f_{1}, f_{2}, \ldots, f_{k}\right.$, $c_{1}, c_{2}, \ldots, c_{c}$ ] including $k$ key functions and $c$ design constraints. The remaining $n$ elements are listed orderly in the PDS vector.

\subsubsection{Generate necessary function-behaviour schemes at system level}

Generate a behaviour space from a multi-disciplinary point of view The rigid key function requirements must be met. Therefore, we need to generate a possible behaviour space from different disciplinary views of discipline-based scientific reasoning, design knowledge and experience and the function-to-behaviour mapping techniques used in the FB-S model [20]. As a result, we can have a possible behaviour space, $B=\left[B_{1}, B_{2}, \ldots, B_{m}\right]$, to respond to key function requirements, $f_{1}, f_{2}, \ldots, f_{k}$.

Given that $B_{1}, B_{2}, \ldots, B_{m}$ are only possible single discipline behaviours, is there any single behaviour good enough to meet all requirements in PDS or do we need to explore multi-disciplinary system function behaviour? To answer these questions, we move to the next step.

Analyse the relationships between the vector $B$ and the vector PDS and establish the mapping $M_{1} M_{1}$ can be structurally viewed and established from design knowledge and experience. Its element $a_{i j}(i=1$ to $n ; j=1$ to $m$ ) describes the correlation relationship (the value is between 0 and 1) between the element $P_{i}$ in $P$ and the element $B_{j}$ in $B$.

$M_{1}=\left[\begin{array}{ccc}a_{11} & \cdots & a_{1 m} \\ \vdots & \ddots & \vdots \\ a_{n 1} & \cdots & a_{n m}\end{array}\right]$

The mapping $M_{1}$ between $P$ and $B$ can be then used for behaviour scheme $\left(B_{\text {scheme }}\right)$ generation and evaluation. The element $B_{j}{ }^{w}$ is the value to reflect on relative correlations to the weighted design requirement space $(P)$.

$$
\begin{aligned}
B_{\text {scheme }} & =W \times M_{1}=\left[w_{1}, w_{2}, \ldots, w_{n}\right] \times\left[\begin{array}{ccc}
a_{11} & \cdots & a_{1 m} \\
\vdots & \ddots & \vdots \\
a_{n 1} & \cdots & a_{n m}
\end{array}\right] \\
& =\left[B_{1}^{w}, B_{2}^{w}, \ldots, B_{m}^{w}\right]
\end{aligned}
$$

The element $B_{j}{ }^{w}$ with the biggest value in $B_{\text {scheme }}$ suggests that the behaviour scheme $B_{j}$ can possibly support the key function requirements and have the best overall support to other design requirements in $P$.

Evaluate the behaviour schemes and identify and create possible system function behaviours After obtaining $B_{\text {scheme, }}$ we can evaluate the corresponding behaviour schemes with the biggest value or the second biggest value in $B_{\text {scheme }}$ against the design constraints, $c_{1}, c_{2}, \ldots, c_{c}$. If all constraints can be met, it means that the single disciplinary behaviour solution is found and then can move on to search its structure mapping $\left(M_{2}\right)$. Here, we do not discuss $M_{2}$ in detail because it is out of research focus. The readers can refer to the reference.

If none of single disciplinary behaviours can meet the design constraints, it suggests that we need to combine two or three single disciplinary behaviours to generate a system function-behaviour scheme, for example, to structurally and behaviourally integrate the best two single discipline behaviour solutions into one system function-behaviour solution (scheme).

At this step, for a combined multi-disciplinary scheme, we can evaluate it, based on our design knowledge and experience, by incorporating DBM analysis, which is detailed in the next step.

\subsubsection{Develop a multi-disciplinary behaviour matrix $\left(M_{3}\right)$ for analysis and evaluation of a SFB scheme}

For a chosen SFB scheme, we need to establish its multidisciplinary behaviour model. For a single disciplinary scheme, its behaviour can be described as a series of state changes in a sequence from $B v_{1}, B v_{2}, \ldots, B v_{n}$ through the state controls. For a multi-disciplinary scheme, when looking at how to make different disciplinary solutions work together, 
we examine how one behaviour from a discipline can couple with the other disciplinary behaviours. We then establish a multi-disciplinary behaviour matrix to explore the possible state and associated control connections. Let states for behaviour $D_{i}=\left\{B v_{1}, \ldots, B v_{n}\right\}$, the states for behaviour $D_{j}=\left\{B v_{m}, \ldots\right.$, $\left.B v_{l}\right\}$, then the corresponding multi-disciplinary behaviour matrix can be described as $M_{3}$ or $D_{i j}$. Its element value could be null to represent no interaction relationship between the two related behaviour states or a possible interaction relationship.

$$
D_{i j}=\begin{array}{c|ccc} 
& B v_{m} & \cdots & B v_{l} \\
\hline B v_{1} & R_{1 m} & \cdots & R_{1 l} \\
\vdots & \vdots & \vdots & \vdots \\
B v_{n} & R_{n m} & \cdots & R_{n l}
\end{array}
$$

Note that if there is another behaviour to be coupled in the behaviour scheme, we just need to add its states to enlarge the top row to $\left\{B v_{m}, \ldots, B v_{l}\right\}$.

To establish this matrix, there is a need for crossdisciplinary team efforts to identify the possible interactions and coupling mechanisms between the concerned states and their controls. This is, in general, an analysis process during the matrix development, but meanwhile, the matrix can be used for evaluating a multi-disciplinary SFB scheme. For example, if no state interaction can be practically found between the two behaviours, they cannot be integrated as a feasible SFB scheme. If there are many possible state interactions, we can continue to examine and evaluate possible structures to support the multi-disciplinary scheme in the next step.

\subsubsection{Develop structure mapping and SFB scheme evaluation}

For a single disciplinary scheme, the mapping between behaviours and realisation structures can be developed from previous design and experiences with discipline design experts.

For a multi-disciplinary scheme, from the identified possible state interactions crossing multi-disciplinary behaviours, we first search for a single disciplinary behaviour-structure mapping and then develop multi-disciplinary coupling structures to bond them together. Again, this needs crossdisciplinary team efforts from both structure and state control points of view to identify possible structure connections as a bridge to integrate single discipline structures. The identifications of the bridge elements will form a mapping matrix $\left(M_{4}\right)$. After that, we can use $M_{4}$ to evaluate the practicability of implementing the bridge. These evaluations can lead to a final P-B-S design scheme selection and then look backwards to evaluate the final P-B-S design scheme for a final conceptual design solution.

\section{Design case study}

In this paper, a high-speed train's braking system is selected for our case study of designing a CoPS. The proposed methods and strategies are applied to obtain the conceptual design solution scheme, and the proposed design model and tools are verified.

\subsection{Problem description}

Braking is one of the most important working conditions of a running train. For a passenger train, its braking system's performance directly affects not only the train's running safety but also its passengers' safety and experience. With the increasing train's running speed, it is necessary to design a braking system, which can provide more powerful braking capability, safety and reliability. However, a train braking system is a typical CoPS, which has diversified functions and complex structure hierarchy. Its working mechanism involves multiple disciplines. Design of a train braking system often needs collaboration with the experts in various disciplines. Therefore, at the conceptual design stage of a train braking system, how to realise rapid and effective mapping from PDS to design schemes is an important problem in design.

\subsection{Problem solution}

\subsubsection{Create a PDS vector}

A train braking system functions when the train needs service braking or emergency braking. The service braking is an action of adjusting and controlling speed in the normal operation of the train, which is relatively moderate and smooth. According to the braking level, braking force can be adjusted and is generally within 20 80 \% capacity of the braking device. Emergency braking is an abnormal action of stopping the train in a very short time in case of accidents and other emergency conditions, which is powerful and sharp. Braking force reaches the full capacity of the braking device in a very short time.

For the conceptual design of a train braking system, we start with constructing a product design specification (or a requirement space) for a braking system as shown in Fig. 3. We divide the critical requirement elements into rigid constraint (function and performance) and goal variables. We determine their degrees of importance by subjective or objective experience.

These requirement elements are listed as the initial input vector $(P)$, with the corresponding weighting vector $(W)$. They are $P=\left[P_{1}, P_{2}, P_{3}, P_{4}, P_{5}, P_{6}, P_{7}, P_{8}, P_{9}\right]$ and $W=\left[w_{1}, w_{2}, w_{3}\right.$, $\left.w_{4}, w_{5}, w_{6}, w_{7}, w_{8}, w_{9}\right]$.

$P_{1}$ and $P_{2}$ represent service braking and emergency braking as functional requirements. $P_{3}, P_{4}$ and $P_{5}$ represent 
Fig. 3 Braking system design requirement space

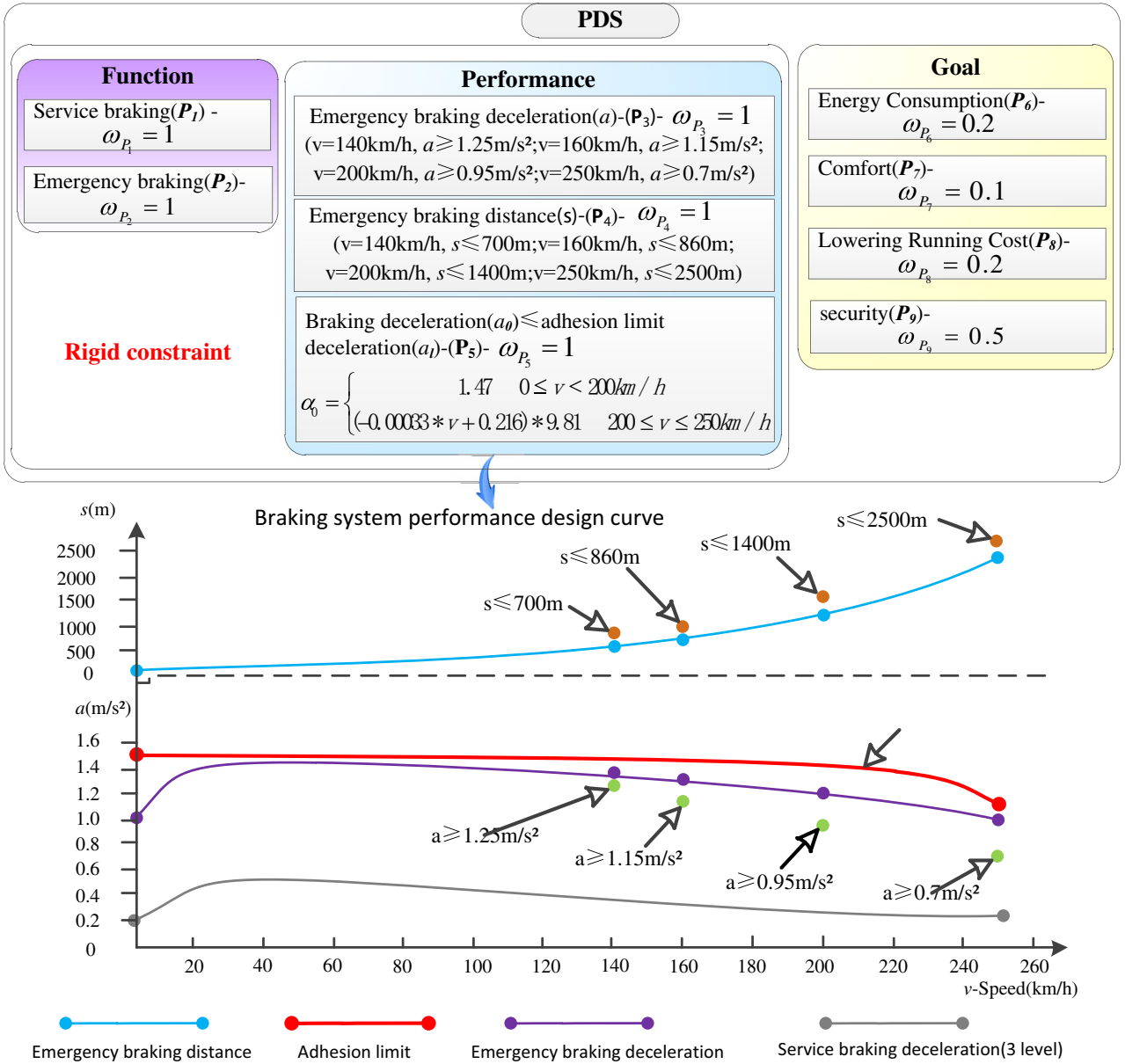

performance constraints: emergency braking deceleration, emergency braking distance and adhesion limit, respectively, while $P_{7}, P_{8}$ and $P_{9}$ indicate other design goals such as comfort, lowering running cost and security requirements, respectively.

The corresponding weight vector $(W)$ is determined by experience as $[1,1,1,1,1,0.2,0.1,0.2,0.5]$.

From Eq. (1), we get PDS $=\left[1 \cdot P_{1}, 1 \cdot P_{2}, 1 \cdot P_{3}, 1 \cdot P_{4}, 1 \cdot P_{5}\right.$, $\left.0.2 \cdot P_{6}, 0.1 \cdot P_{7}, 0.2 \cdot P_{8}, 0.5 \cdot P_{9}\right]$. The weights for parameters $P_{1}$, $P_{2}, P_{3}, P_{4}$ and $P_{5}$ are 1, which indicates that these PDS parameters are rigid constraints and directly impact on the whole conceptual design process. The conceptual design of braking system must meet the function requirements and performance constraints. After splitting those rigid constraints, the remaining elements listed orderly in the PDS vector are PDS $'=\left[0.5 \cdot P_{9}, 0.2 \cdot P_{6}, 0.2 \cdot P_{8}, 0.1 \cdot P_{7}\right]$; that is, the braking system should first meet the security requirements $\left(P_{9}\right)$ and then energy consumption $\left(P_{6}\right)$ or lowering running $\operatorname{cost}\left(P_{8}\right)$ and comfort $\left(P_{7}\right)$.

Before considering what functional behaviour schemes generate braking force, we first check the performance constraints from PDS and produce the deceleration curve of braking system as shown in Fig. 3. Firstly, the deceleration curve (in pink) of emergency braking is designed to meet the requirements of emergency braking deceleration in the PDS and, under the adhesion limit line (in red), the emergency braking distance that resulted from the deceleration curve also needs to meet the requirements of the emergency braking distance (shown in blue line). Secondly, the service braking deceleration curve (in grey) is designed under the emergency braking deceleration curve (in pink) and, normally, there are seven levels in the service braking with the corresponding deceleration curves. Figure 3 shows the third-level service braking deceleration curve (in grey).

\subsubsection{Generate necessary function-behaviour schemes at system level}

Braking force can be generated in several ways, including electromagnetic braking (magnetic track braking $\left(B_{1}\right)$, eddy current braking $\left(B_{2}\right)$ ), pneumatic braking (tread brake $\left(B_{3}\right)$, disc braking $\left(B_{4}\right)$ ) and electric braking $\left(B_{5}\right)$. According to the design experience, the mapping relationship matrix $\left(M_{1}\right)$ is determined between five functional schemes $\left(B_{1}, B_{2}, B_{3}, B_{4}\right.$ and $\left.B_{5}\right)$ and PDS goal elements $\left(P_{9}, P_{6}, P_{8}\right.$ and $\left.P_{7}\right)$, as follows. 


$M 1=$\begin{tabular}{r|ccccc} 
& $B_{1}$ & $B_{2}$ & $B_{3}$ & $B_{4}$ & $B_{5}$ \\
\hline$P_{9}$ & 0.3 & 0.3 & 0.9 & 0.9 & 0.3 \\
$P_{6}$ & 0.3 & 0.3 & 0.1 & 0.3 & 0.9 \\
$P_{8}$ & 0.1 & 0.1 & 0.1 & 0.3 & 0.9 \\
$P_{7}$ & 0.3 & 0.3 & 0.1 & 0.3 & 0.3
\end{tabular}

The coupling relationship ranges from 0.1 to 0.9 . The strength of the relationship is classified as follows: strong correlation $=0.9$, moderate correlation $=0.3$ and weak correlation $=0.1$.

Finally, by applying a matrix operation from PDSbehaviour mapping (Eq. (3)), we get the result $B_{\text {scheme }}=[0.26,0.26,0.5,0.6,0.54]$.

From the result, it can be seen that the pneumatic disc braking scheme $\left(B_{4}\right)$ is most suitable for goal requirements. The pneumatic disc braking scheme produces the braking force by friction, and the kinetic energy is converted into heat energy to realise the train braking, but this method wears the brake disc and brake pad seriously and increases the energy consumption and operation cost. Under the condition of train emergency braking, the pneumatic disc braking is a safe and reliable method. However, under the condition of service braking, considering the cost and energy consumption requirements, it is required to choose another behaviour way from $B$ to help realise the service braking function of the train. The electric braking scheme $\left(B_{5}\right)$ is the second suitable candidate in $B$. Therefore, we examine the electric braking scheme $\left(B_{5}\right)$ for possible integration.

Electric braking is to convert kinetic energy into electric energy, which can provide a large braking force with low energy consumption and low operation cost. However, because the electric braking cannot provide braking force in case of the loss of electricity supply, safe parking cannot be achieved in an emergency condition (such as the loss of electricity), which is also the reason why pneumatic braking is essential. Furthermore, in the low speed stage $(0-15 \mathrm{~km} / \mathrm{h})$, the phenomenon of the magnetic induction line is weakened or even disappeared because of the decrease of the speed, which makes the loss of electric braking force, and electric braking force cannot meet the braking requirements of the low speed range. In order to realise the service braking function of the train, we choose the electric braking $\left(B_{5}\right)$ as the main mode and then select the pneumatic disc braking $\left(B_{4}\right)$ to complement the braking function of the train in the low speed range and in case of losing power. Thus, we design the high-speed train's braking system by integrating pneumatic braking and electric braking, termed as the electric pneumatic braking scheme, which relates to the mechanical, pneumatic, electric, control and other disciplines, thus forming a multi-disciplinary system behaviour scheme.

\subsubsection{Develop multi-disciplinary behaviour matrix $\left(M_{3}\right)$ for analysis and evaluation of a SFB scheme}

The electric pneumatic braking scheme includes two kinds of braking behaviours: the one is to use air pressure to press the brake pad on the brake disc to get the required braking force and the other is to transform the traction motor into a generator so that the kinetic energy of the train is converted into electrical energy. The design of the braking system needs to consider the collaborative design of various disciplines with different physical principles. It includes four disciplines: the control, electrical, mechanical, and pneumatic, and their disciplinary behaviour parameters are interrelated. For example, different braking levels lead to different decelerations, and in turn, different decelerations require the corresponding braking forces based on vehicle weight. In addition, the air braking force is not only affected by the electric braking capability but also the brake pad clamping force of mechanical discipline and the brake cylinder pressure of the pneumatic discipline. They all change along with the change of deceleration level of control discipline. Figure 4 shows each disciplinary behaviour, behaviour parameters and the relationship between these parameters.

From Fig. 4, we build the multi-disciplinary behaviour matrix $\left(M_{4}\right)$ of control, electric, mechanical and pneumatic disciplines with reference to Eq. (4).

\begin{tabular}{|c|c|c|c|c|c|c|c|c|c|c|}
\hline & & & $\begin{array}{l}D_{1} \\
B v_{1}\end{array}$ & $B v_{2}$ & $B v_{3}$ & $\begin{array}{l}D_{2} \\
B v_{4}\end{array}$ & $B v_{5}$ & $\begin{array}{l}D_{3} \\
B v_{6}\end{array}$ & $B v_{7}$ & $\begin{array}{l}D_{4} \\
B v_{8}\end{array}$ \\
\hline & & $B v_{1}$ & $x$ & $R_{12}$ & $x$ & $x$ & $x$ & $x$ & $x$ & $x$ \\
\hline & & $B v_{2}$ & $R_{21}$ & $x$ & $R_{23}$ & $x$ & $x$ & $x$ & $x$ & $x$ \\
\hline & & $\mathrm{Bv}_{3}$ & $x$ & $R_{32}$ & $x$ & $x$ & $D R_{35}$ & $x$ & $x$ & $D R_{38}$ \\
\hline & & $B v_{4}$ & $x$ & $x$ & $x$ & $x$ & $R_{45}$ & $\times$ & $D R_{47}$ & $\times$ \\
\hline & & $B v_{5}$ & $x$ & $x$ & $D R_{53}$ & $R_{54}$ & $x$ & $x$ & $x$ & $D R_{5}$ \\
\hline & & $B v_{6}$ & $\times$ & $x$ & $\bar{x}$ & $x$ & $\times$ & $x$ & $R_{67}$ & $\times$ \\
\hline & & $B v_{7}$ & $x$ & $x$ & $x$ & $D R_{74}$ & $x$ & $R_{76}$ & $x$ & $x$ \\
\hline & & $B v_{8}$ & $\times$ & $x$ & $D R_{83}$ & $\bar{x}$ & $D R_{85}$ & $x$ & $\times$ & $\times$ \\
\hline
\end{tabular}


Fig. 4 Hierarchy relationship diagram of multi-disciplinary behaviours of electric pneumatic braking

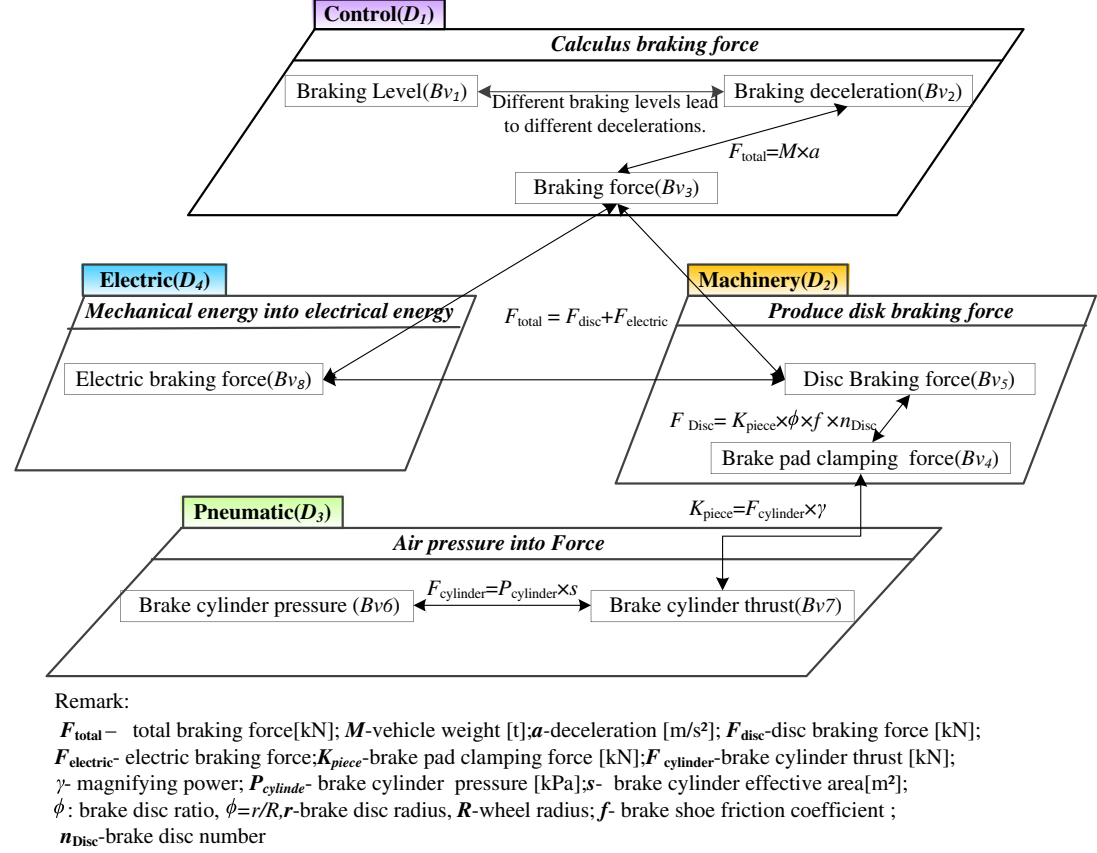

The relationships of disciplinary behaviour parameters are shown in $M_{3}$. In control discipline $\left(D_{1}\right)$, mechanical discipline $\left(D_{2}\right)$ and electrical discipline $\left(D_{4}\right)$, there are coupling relationships among the three behaviour parameters $B v_{3}, B v_{5}$ and $B v_{8}$, such as $D R_{35}, D R_{53}, D R_{38}, D R_{83}, D R_{58}$ and $D R_{85}$, that is $F_{\text {total }}=F_{\text {disc }}+F_{\text {electric. }}$. In mechanical discipline $\left(D_{2}\right)$ and electrical discipline $\left(D_{4}\right)$, there are coupling relationships between behaviour parameters $B v_{4}$ and $B v_{7}$, such as $D R_{47}$ and $D R_{74}$, that is $K_{\text {piece }}=F_{\text {cylinder }} \times \gamma$. Therefore, the electric pneumatic braking is a feasible method for the multi-disciplinary system behaviour scheme, and then we can find the multi-disciplinary structure scheme to realise it.

\subsubsection{Develop multi-disciplinary behaviours to structure mapping and analysis}

According to the DBM or $M_{3}$, we find that the behaviour parameters cross disciplines are influenced by each other, and these behaviours need proper structure as a carrier to achieve. Mapping from behaviours to macrostructures is the qualitative analysis of behaviours, such as brake cylinder thrust, which needs brake cylinder to receive compressed air through compressor, and then compressed air makes the piston movement to produce cylinder thrust. In addition, the production of disc braking force needs a brake disc as a clamping object of the brake pad to achieve the braking function of the train by the friction effect in which power is converted to heat energy. Therefore, there is a need of multi-disciplinary structures including air compressor, brake cylinder, piping, brake pad, brake disc and other accessories work together to achieve the multi-disciplinary behaviours. First, we explore the single discipline structures under the functional structures: brake pad, brake disc, brake force amplifier and brake cylinder (see Fig. 5). Their relationships with behaviours in terms of disc braking force, brake pad clamping force, brake cylinder thrust and brake cylinder air pressure are then identified for developing multi-disciplinary coupling structures. Here, for example, a brake force amplifier is a bridge structure between disc braking force and brake clamping force. Figure 5 is a graphical representation of the corresponding matrix $M_{4}$.

Choosing different structural types and using different structural parameters will produce different behaviour structural solutions. In addition to qualitative analysis of the behaviour structure in the conceptual design phase, the relationships between behaviour parameters and structural parameters is established based on relevant physical principles and they are then used to form a quantitative analysis of different behaviour-structure mapping.

In this case study, we take the braking performance analysis as an example and focus on the mechanical and pneumatic discipline couplings with given control discipline constraints: emergency braking deceleration and braking force. In other words, we do not discuss the coupled control structure here. As shown in Fig. 5, after selecting single discipline structures, the relationships between the structure parameters and behaviour parameters can be illustrated in Fig. 6. Its key structure parameters include brake disc friction radius $(r)$, brake shoe friction coefficient $(f)$, magnification power $(\gamma)$ and brake cylinder effective area $(s)$. The structure schematic diagram of the train bogie with brake rigging is shown in Fig. 7.

According to Fig. 6 and relevant physical principles, we can find the relationships between the behaviour parameters and the structural parameters. It is worth noting that the structural parameters are not set at random because they are often 
Fig. 5 Behaviour parameter mapping to different structures

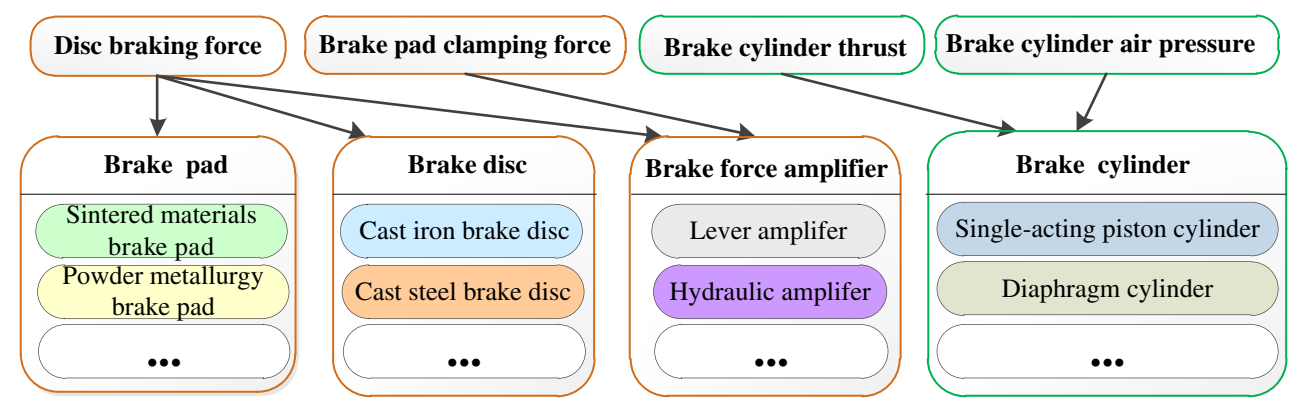

restricted by installation space or its structural design. For instance, the braking structures of the brake system are installed on the bogie and the installation space on the bogie is very limited. Therefore, some implicit structure constraints exist. For example, the number of brake disc cannot be arbitrarily increased and disc radius cannot be greater than the radius of the wheels. In order to effectively use the space, the number of brake discs is generally 8 .

Table 1 shows the analysis results for four design schemes with the corresponding choices of the structures and assignments of structural parameters. All schemes must meet the requirements of braking deceleration $\left(B v_{2}\right)$ and disc braking force $\left(B v_{5}\right)$ from the control and mechanical discipline, which are the requirements of braking performance. In addition, the brake cylinder pressure $\left(B v_{6}\right)$ is a key indicator of evaluating the electric pneumatic braking and the pressure of the total air duct, which is usually controlled within $850-1000 \mathrm{kPa}$. In order to ensure the safety of the train braking, the general requirement for the brake cylinder pressure is less than $600 \mathrm{kPa}$.

Scheme 1 In order to meet the requirements of the train disc braking force and deceleration, the pressure of brake cylinder reaches $4829 \mathrm{kPa}$ in the absence of any measures of increasing the brake cylinder thrust, which is obviously not satisfied with the requirement that the brake cylinder pressure should be less than $600 \mathrm{kPa}$, so scheme 1 is obviously not desirable.
Scheme 2 According to scheme 1, to meet the train braking performance requirements, it is not advisable to rely on increasing the brake cylinder pressure to increase the brake cylinder thrust. We need to take other ways to increase the brake cylinder thrust to meet the braking performance of the train. As shown in Fig. 5, we increase the brake clamping force by the way of leverage and hydraumatic. In scheme 2 , we choose the lever-type brake clamp to increase the brake cylinder thrust, and it is found that the brake cylinder pressure decreases significantly to $604 \mathrm{kPa}$ by calculating, but the scheme is still not ideal.

Scheme 3 According to scheme 2, the lever-type brake clamp can increase the thrust of the brake cylinder. However, the number of levers cannot be increased arbitrarily and is subjected to space constraints. Now looking at the relationship matrix of behaviour structure again, we find that the increasing friction radius of the brake disc and the friction coefficient of the brake shoe within the scope of design constraints can increase the braking force of the target disc as well. So in scheme 3, we try to increase the disc radius from 275 to $295 \mathrm{~mm}$ and change the sintering material brake pads into powder metallurgy brake pads to improve the friction coefficient, and the brake cylinder pressure reduces to $483 \mathrm{kPa}$ by calculating, which meets the braking requirements at the same time, so the scheme is a feasible solution.
Fig. 6 Behaviour parameterstructural parameter relationship

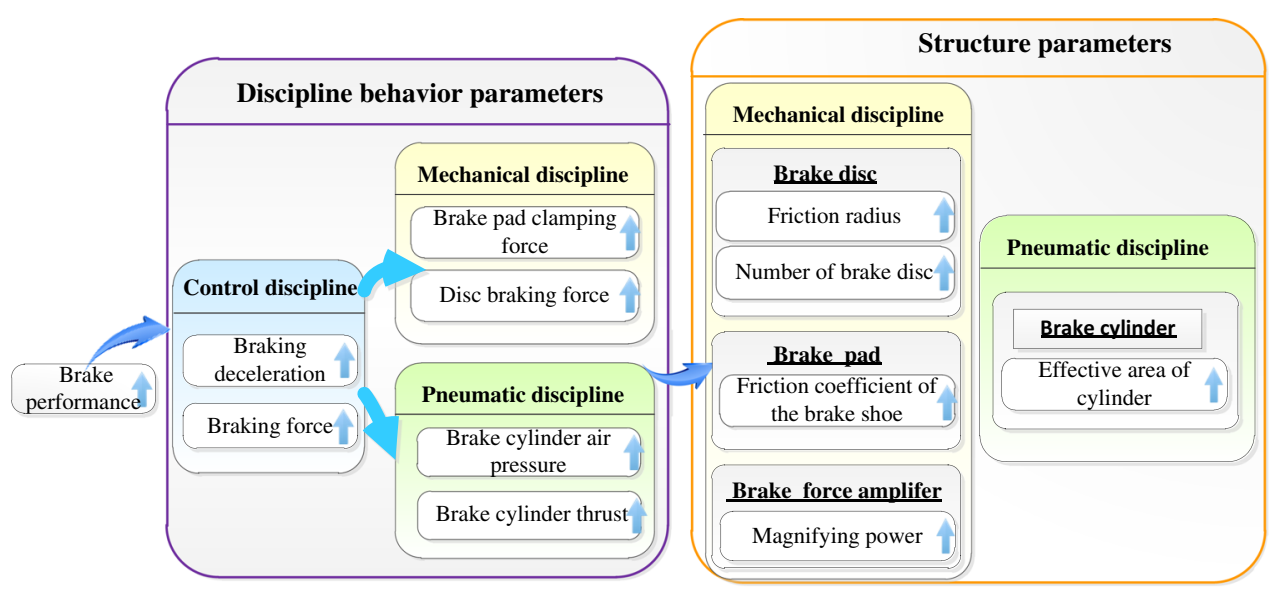


Fig. 7 Structure schematic diagram of the brake rigging on bogie

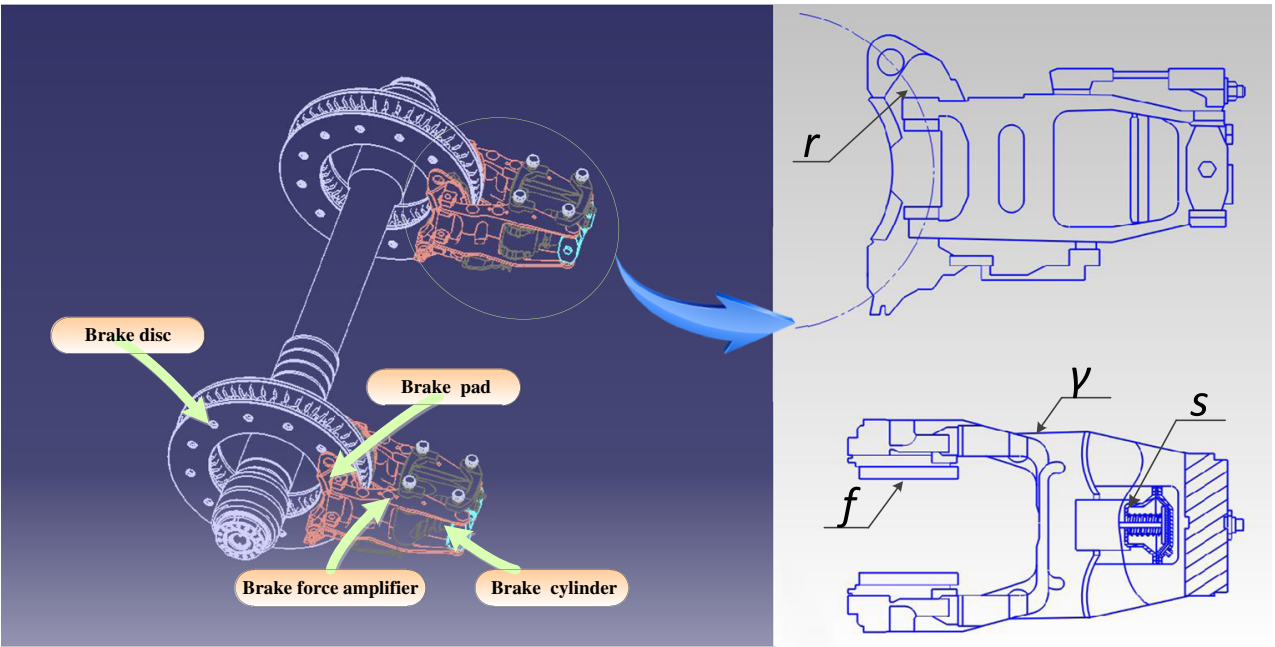

Scheme 4 According to Fig. 5, in addition to the lever-type braking clamp used in scheme 3 , hydraulic braking clamp can also increase the brake cylinder thrust. Its advantages could include small footprint, large multiplication of rigging and rapid response, but its disadvantages include oil leaking and the need of a cleaning device. On the basis of scheme 3 , the parameters of the brake disc and brake pad are not changed and the hydraulic braking clamp is used in scheme 4 . Brake cylinder pressure is only $318 \mathrm{kPa}$ in scheme 4 , which is the smallest of all the schemes, and it is a feasible solution.

In conclusion, schemes 1 and 2 do not meet the design requirements because they are not feasible. Schemes 3 and 4 are both feasible solutions, since the brake cylinder pressure of scheme 4 is smaller, so the scheme 4 is the final solution (optimal solution).

\section{Discussion}

In the case study examined, a key observation that can be made about the approach is that we can quickly and effectively obtain an optimal scheme by this method in the conceptual design phase. We realise that the premise of the conceptual design of a high-speed train's braking system is assuming its design knowledge and experience that are known.
The design knowledge and experience are expressed as elements in the mapping matrices from $W$ to $M_{1}, M_{3}$ and $M_{4}$. The accuracy of design knowledge and experience expression is important because they affect design scheme selections and evaluations, but it is difficult to guarantee. On the other hand, our design approach makes the use of design knowledge practical because we decompose and embed overall highly nonlinear-coupled design knowledge from multidisciplines into well-structured and understandable matrices from $W$ to $M_{1}, M_{3}$ and $M_{4}$. The advantage of organising the existing design experience and knowledge in the matrices is to realise knowledge reuse and capturing and, finally, to further evaluate and form a standardised, formalised complex product conceptual representation and evaluation tool. However, a high-speed train's braking system is a complex multi-disciplinary system and its design involves knowledge coupling of different disciplines such as in the establishment of matrix $M_{4}$. Therefore, multi-disciplinary team efforts are required to make our approach workable. In general, in the early stage of the development of CoPS, if design knowledge and experience are available or even triable, this method is feasible to get the best solution, which seizes the main contradiction to form a number of feasible solutions, and applies the matrix conversion and calculation to evaluate the solution.

Table 1 Behaviour structure schemes

\begin{tabular}{|c|c|c|c|c|c|c|c|c|c|c|c|}
\hline & \multicolumn{3}{|c|}{ Design requirements } & \multicolumn{2}{|c|}{ Brake disc $U\left(S_{1}\right)$} & \multirow{2}{*}{$\begin{array}{l}\text { Brake pad } U\left(S_{2}\right) \\
f\end{array}$} & \multicolumn{2}{|c|}{ Brake clamp $U\left(S_{3}\right)$} & \multicolumn{3}{|c|}{ Analysis results } \\
\hline & $B v_{2}$ & $B v_{5}$ & $B v_{6}$ & $n_{\text {disc }}$ & $r$ & & $s$ & $\gamma$ & $B v_{2}$ & $B v_{5}$ & $B v_{6}$ \\
\hline Scheme 1 & 1.25 & 85 & $<600$ & 8 & 275 & 0.3 (sintered materials) & 110 & 1 & 1.25 & 85 & 4829 \\
\hline Scheme 2 & 1.25 & 85 & $<600$ & 8 & 275 & 0.3 (sintered materials) & 110 (lever) & 8 & 1.25 & 85 & 604 \\
\hline Scheme 3 & 1.25 & 85 & $<600$ & 8 & 295 & 0.35 (powder metallurgy) & 110 (lever) & 8 & 1.25 & 85 & 483 \\
\hline Scheme 4 & 1.25 & 85 & $<600$ & 8 & 295 & 0.35 (powder metallurgy) & 18 (hydraulic) & 74 & 1.25 & 85 & 318 \\
\hline
\end{tabular}




\section{Conclusions}

In this paper, a new conceptual design method for CoPS with an effective mapping tool has been proposed, which is significant for the automation of conceptual design process and consistent management of design information in the field of engineering design. Based on the proposed P-B-S concept design model, three vector-based mapping tools are developed as a framework to gain insights of multi-disciplinary relations, guide the coupling PDS-behaviour-structure searching and generate/evaluate conceptual design schemes. This method can be used in multi-disciplinary engineering design projects with some domain knowledge support.

Our case study of high-speed train's braking system design shows that (1) the P-B-S conceptual design model is feasible and applicable in multi-disciplinary engineering design and (2) the proposed method is a useful tool to realise the rapid and effective mapping from PDS to conceptual design schemes.

As demonstrated in the case study, the implementation of the proposed design method requires some disciplinary design knowledge support. Therefore, in the future, a conceptual design knowledge base could be established to support the computerisation of the conceptual design method.

Acknowledgments This work was partially supported by the National Natural Science Foundation of China (Grant Nos. 51305367 and 51575461). It is also supported by the Doctoral Student Innovation Funds for Zhang Haizhu from Southwest Jiaotong University.

Open Access This article is distributed under the terms of the Creative Commons Attribution 4.0 International License (http:// creativecommons.org/licenses/by/4.0/), which permits unrestricted use, distribution, and reproduction in any medium, provided you give appropriate credit to the original author(s) and the source, provide a link to the Creative Commons license, and indicate if changes were made.

\section{References}

1. Pahl G, Beitz W (2013) Engineering design: a systematic approach [M]. Springer Science \& Business Media

2. Qiu SL, Fok SC, Chen CH et al (2002) Conceptual design using evolution strategy. Int J Adv Manuf Technol 20(9):683-691

3. Hsu W, Woon IMY (1998) Current research in the conceptual design of mechanical products. Comput Aided Des 30(5):377-389

4. Finke RA, Ward TB, Smith SM (1992) Creative cognition: theory, research, and applications

5. Forsberg K, Mooz H (1992) The relationship of systems engineering to the project cycle. Eng Manag J 4(3):36-43

6. Hobday M (1998) Product complexity, innovation and industrial organisation. Res Policy 26(6):689-710, Advanced Engineering Informatics, 2012, 26(2): 292-305

7. Bin H, Feng LH, Zhen LW et al (2010) Automated conceptual design of multiple input and multiple output mechanical transmission system. Computer Engineering and Applications (ICCEA), 2010 Second International Conference on IEEE 2:78-82
8. Suh NP (2001) Axiomatic design: advances and applications. The Oxford Series on Advanced Manufacturing

9. Camelo DM, Mulet E (2010) A multi-relational and interactive model for supporting the design process in the conceptual phase. Autom Constr 19(7):964-974

10. Chen Y, Liu ZL, Xie YB (2012) A knowledge-based framework for creative conceptual design of multi-disciplinary systems. Comput Aided Des 44(2):146-153

11. Pahl G, Beitz W (1984) Engineering design the design council. Springer, London

12. Avgoustinov N (2007) Modelling in mechanical engineering and mechatronics: towards autonomous intelligent software models. Springer Science \& Business Media

13. Chandrasegaran SK, Ramani K, Sriram RD et al (2013) The evolution, challenges, and future of knowledge representation in product design systems. Comput Aided Des 45(2):204-228

14. Christophe F, Bernard A, Coatanéa É (2010) RFBS: a model for knowledge representation of conceptual design. CIRP Ann Manuf Technol 59(1):155-158

15. Chang XM, Sahin A, Terpenny J (2008) An ontology-based support for product conceptual design. Robot Comput Integr Manuf 24(6):755-762

16. Finger S, Dixon JR (1989) A review of research in mechanical engineering design. Part 1: descriptive, prescriptive, and computerbased models of design process. Res Eng Des 1(1):51-67

17. Cross N (1997) Descriptive models of creative design: application to an example. Des Stud 18(4):427-455

18. Deng YM, Zhu YW (2009) Function to structure/material mappings for conceptual design synthesis and their supportive strategies. Int J Adv Manuf Technol 44(11-12):1063-1072

19. Umeda Y, Ishii M, Yoshioka M et al (1996) Supporting conceptual design based on the function-behaviour-state modeler. Artificial Intelligence in Engineering Artificial Intelligence for Engineering, Design, Analysis and Manufacturing 10(4):275-288

20. Gero JS, Kannengiesser U (2004) The situated function-behaviour-structure framework. Des Stud 25(4):373-391

21. Deng YM (2002) Function and behaviour representation in conceptual mechanical design. Artificial Intelligence for Engineering Design, Analysis and Manufacturing 16(5):343-362

22. Chen Y, Zhao M, Xie Y et al (2015) A new model of conceptual design based on scientific ontology and intentionality theory. Part II: the process model. Des Stud 38:139-160

23. Stone RB, Wood KL (2000) Development of a functional basis for design. J Mech Des 122(4):359-370

24. Nage RL, Perry KL, Stone RB et al (2009) Function CAD: a functional modeling application based on the function design framework. Proceedings of the ASME 2009 International Design Engineering Technical Conferences \& Computers and Information in Engineering Conference IDETC/CIE, California

25. Karniela A, Reicha Y (2013) Multi-level modelling and simulation of new product development processes. J Eng Des 24(3):185-210

26. Ölvander J, Lundén B, Gavel H (2009) A computerized optimization framework for the morphological matrix applied to aircraft conceptual design. Comput Aided Des 41(3):187-196

27. Park T, Kim KJ (1998) Determination of an optimal set of design requirements using house of quality. J Oper Manag 16(5):569-581

28. Le Q, Panchal JH (2012) Analysis of the interdependent coevolution of product structures and community structures using dependency modelling techniques. J Eng Des 23(10-11):807-828

29. Yang B, Wang W, Li HT (2010) Product design based on biological growth mechanism. 2010 International Conference on Intelligent System Design and Engineering Application, Changsha, China, pp 13-14

30. Kolodner J (2014) Case-based reasoning. Morgan Kaufmann 
31. Wang L, Shen W, Xie H et al (2002) Collaborative conceptual design — state of the art and future trends. Comput Aided Des 34(13):981-996

32. Wang YX, Yan HS (2002) Computerized rules-based regeneration method for conceptual design of mechanisms. Mech Mach Theory 37(9):833-849

33. Skoutas D, Simitsis A (2007) Ontology-based conceptual design of ETL processes for both structured and semi-structured data. International Journal on Semantic Web and Information Systems 3(4): $1-24$

34. Huang HZ, Bo R, Chen W (2006) An integrated computational intelligence approach to product concept generation and evaluation. Mech Mach Theory 41(5):567-583

35. Strawbridge Z, McAdams DA, Stone RB (2002) A computational approach to conceptual design. ASME 2002 International Design Engineering Technical Conferences and Computers and Information in Engineering Conference. American Society of Mechanical Engineers. p. 15-25

36. Bryant CR, Stone RB, McAdams DA et al (2005) Concept generation from the functional basis of design. International conference on engineering design ICED 05, Melbourne, Australia

37. Cardillo A, Cascini G, Frillici F (2011) Computer-aided embodiment design through the hybridization of mono objective optimizations for efficient innovation process. Comput Ind 62(4):384-397

38. Komoto H, Tomiyama T (2010) Computational support for system architecting. Proceedings of the ASME 2010 International Design Engineering Technical Conferences \& Computers and Information in Engineering Conference IDETC/CIE, Quebec, Canada

39. Zhang WY, Tor SB, Britton GA (2001) A prototype knowledgebased system for conceptual synthesis of the design process. Int $\mathrm{J}$ Adv Manuf Technol 17(8):549-557

40. D'Amelio V, Chmarra MK, Tomiyama T (2011) Early design interference detection based on qualitative physics. Res Eng Des 22(4): 223-243

41. Tilstra AH, Seepersad CC, Wood KL (2012) A high-definition design structure matrix (HDDSM) for the quantitative assessment of product architecture. J Eng Des 23(10-11):767-789

42. Hehenberger P, Poltschak F, Zeman K et al (2010) Hierarchical design models in the mechatronic product development process of synchronous machines. Mechatronics 20(8):864-875

43. Hehenberger P (2014) Perspectives on hierarchical modeling in mechatronic design. Adv Eng Inform 28(3):188-197

44. Komoto H, Tomiyama T (2012) A framework for computer-aided conceptual design and its application to system architecting of mechatronics products. Comput Aided Des 44(10):931-946 